\title{
Y-27632 inhibits ethanol-induced increase in intestinal epithelial barrier permeability
}

\author{
JING TONG, YING WANG, BING CHANG, DAI ZHANG and BINGYUAN WANG \\ Department of Gastroenterology, The First Affiliated Hospital of China Medical University, \\ Shenyang, Liaoning 110001, P.R. China
}

Received October 10, 2013; Accepted February 26, 2014

DOI: $10.3892 / \mathrm{mmr} .2014 .2060$

\begin{abstract}
The present study aimed to investigate the effect of Y-27632, an inhibitor of the Rho-associated protein kinase (ROCK), which belongs to a family of downstream effectors of activated RhoA, on the ethanol-induced increase in permeability of the intestinal epithelial barrier (IEB). The in vitro model of IEB was established usiung Caco-2 cells, and the cells were pretreated with Y-27632 prior to treatment with ethanol for $60 \mathrm{~min}$. Transepithelial resistance (TEER) and paracellular marker Lucifer yellow flux measurements were performed to assess the IEB permeability. The localization and expression of tight junction (TJ)-associated proteins were detected by immunofluorescence and western blot analysis, respectively. Y-27632 partially inhibited epithelial leakage and restored normal TEER values in the IEB. Immunofluorescence and western blot analysis results indicated that ethanol induces a shift from the insoluble to the soluble fractions of claudin-1, and that the ethanol-induced decreased expression of the zonula occludens-1 (ZO-1) protein is restored by Y-27632. In conclusion, our results suggest that ROCK may play a key role in the ethanol-induced increase of IEB permeability.
\end{abstract}

\section{Introduction}

Alcoholic liver disease (ALD) remains an important cause of morbidity and mortality, with >75,000 annual deaths worldwide and incidence rates having increased in the last decade $(1,2)$. ALD encompasses a spectrum of pathological states including fatty liver, inflammation, fibrosis, cirrhosis, and even malignancy $(3,4)$. Clinical and experimental data have demonstrated that gut-derived endotoxins and endotoxemia play a major role in the development of $\operatorname{ALD}(5,6)$.

Ethanol can affect the intestinal barrier in multiple ways, leading to an increased portal blood level of endotoxin and

Correspondence to: Dr Bingyuan Wang, Department of Gastroenterology, The First Affiliated Hospital of China Medical University, 155 North Nanjing Street, Shenyang, Liaoning 110001, P.R. China

E-mail: wangby_doctor@163.com

Key words: ROCK, ethanol, intestinal epithelial barrier, permeability, tight junctions hepatic exposure, thereby further promoting liver inflammation and the progression to ALD (7). Numerous studies have indicated that ethanol induces intestinal permeability in vivo via oxidative stress $(7,8)$. Antioxidants have been shown to normalize intestinal permeability in alcoholic patients (9). It is now generally accepted that impaired intestinal epithelial integrity and intestinal barrier dysfunctions are the two fundamental causes of increased intestinal permeability $(10,11)$. The intestinal epithelial barrier is a complex system composed of cellular, physical, and chemical components (12). The epithelial cells form a layer with the paracellular space sealed by tight junctions (TJs) and adherens junctions (AJs). TJs are essential in maintaining intestinal mucosal integrity, which can effectively prevent bacteria, endotoxins and other harmful substances from entering into the blood stream through the intestinal barrier (13). Two studies indicated that altered expression of the TJ-associated proteins zonula occludens-1 (ZO-l) and claudin-1 associates with increased intestinal permeability and higher susceptibility to ALD $(14,15)$. Most studies to date have addressed the potential effects of ethanol on paracellular permeability and disruption of epithelial TJs in a cell culture model of intestinal epithelium, the Caco- 2 cell monolayer (16-18).

The Rho-associated protein kinase (ROCK) is a serinethreonine kinase that acts as a key downstream effector of RhoA signaling, and was shown to contribute in the maintenance of TJ integrity in endothelial cells (19) and in human intestinal epithelial cells (20). In this study, we investigated the hypothesis that ROCK plays a key role in the ethanolinduced increase in permeability of the intestinal epithelial barrier (IEB), and investigated the molecular links between TJ-associated proteins, IEB permeability and ROCK.

\section{Materials and methods}

Caco-2 cells culture and establishment of an in vitro IEB model. The human colon adenocarcinoma cell line Caco-2 was obtained from the American Type Culture Collection (Manassas, VA, USA). Caco-2 cells were cultured in HyClone ${ }^{\mathrm{TM}}$ Dulbecco's modified Eagle's medium (DMEM) purchased from Thermo Fisher Scientific (Waltham, MA, USA) and supplemented with $4.5 \mathrm{mg} / \mathrm{ml}$ glucose, $50 \mathrm{U} / \mathrm{ml}$ penicillin, $50 \mathrm{U} / \mathrm{ml}$ streptomycin, $4 \mathrm{mM}$ glutamine, and $10 \% \mathrm{HyClone}^{\mathrm{TM}}$ fetal calf serum (FCS), in a humidified atmosphere (95\% air, 
$5 \% \mathrm{CO}_{2}$ ) at $37^{\circ} \mathrm{C}$. To establish an in vitro intestinal epithelial barrier model, Caco-2 cells were plated onto Transwell filters (Corning, New York, NY, USA) and regularly monitored by visual inspections under an inverted microscope and by measurements of epithelial resistance.

Assessment of intestinal epithelial barrier permeability. Measurements of transepithelial electrical resistance (TEER) and Lucifer yellow (LY) flux were performed to assess the permeability of IEB. The electrical resistance of Caco-2 cell monolayers cultured on Transwell filters was measured using a Millicell ${ }^{\circledR}$-ERS instrument (Millipore, Bedford, MA, USA). Electrical resistance of Transwell insert was expressed in units of ohm $(\Omega) / \mathrm{cm}^{2}$. The Lucifer yellow fluorescent dye (SigmaAldrich, St. Louis, MO, USA) was added to the upper chamber of the Transwell system at $40 \mu \mathrm{g} / \mathrm{ml}$ in serum-free DMEM. Following treatment with ethanol for different time periods, the medium from the lower chamber was collected. The absorbance of the medium was measured on a fluorescence spectrophotometer (excitation wavelength $427 \mathrm{~nm}$, emission wavelength $536 \mathrm{~nm}$ ), and the LY concentration was calculated based on a standard curve. The LY flux rate $(\%)=$ LY concentration in the lower chamber/LY concentration in the upper chamber.

Drug treatment and experimental groups. First, Caco-2 cells were plated onto the lower side of the Transwell insert and were grown in DMEM. When $80 \%$ of Caco- 2 cells were confluent (at $\sim 21-23$ days of culture), they were seeded on the upper chamber of the Transwell insert, and ethanol was added. The cultured cells were then rinsed with cold phosphatebuffered saline (PBS). Subsequently, Caco-2 cells from the upper chamber were harvested by gently scraping with a cell scraper and were stored at $-80^{\circ} \mathrm{C}$. We previously showed that ethanol treatment causes a concentration- and time-dependent decrease in Caco-2 transepithelial resistance and an increase in the transepithelial permeability to the paracellular marker LY. We also demonstrated that IEB permeability reaches a peak at $60 \mathrm{~min}$ of treatment with $5 \%$ ethanol (21). Based on these findings, the present experimental plan included 6 groups ( $n=4 /$ group): the control (monolayer of Caco- 2 cells), the ethanol (5\%) 0-min group, the ethanol (5\%) 15-min group, the ethanol (5\%) 30-min group, the ethanol (5\%) 60-min group, and the ethanol (5\%) 60-min + Y-27632 group, where Caco-2 cells were pretreated with $10 \mu \mathrm{M}$ Y-27632 for $60 \mathrm{~min}$, then treated with ethanol for $60 \mathrm{~min}$.

Western blot analysis. The Caco-2 cells were washed three times with Dulbecco's phosphate-buffered saline (D-PBS) containing $0.1 \mathrm{mM}$ ethylenediamine tetraacetic acid (EDTA) without calcium and magnesium; then, they were homogenized in $1 \mathrm{ml}$ lysis buffer A [ $2 \mathrm{mM}$ EDTA, $10 \mathrm{mM}$ ethylene glycol tetraacetic acid (EGTA), $0.4 \%$ sodium fluoride (NaF), $20 \mathrm{mM}$ Tris-HCL, protease inhibitor cocktail, phosphatase inhibitor $1 \%$ Triton X-100, pH 7.5] at $4^{\circ} \mathrm{C}$. Samples were centrifuged at $14,000 \mathrm{x} g$ for $30 \mathrm{~min}$, and the supernatant was transferred to a separate tube and collected as the soluble fraction (S). Buffer A $(150 \mu \mathrm{l})$ with $1 \%$ sodium dodecyl sulfate (SDS) at $4^{\circ} \mathrm{C}$ was then added to the pellet. The pellet was disrupted with an ultrasonic crusher. Samples were then centrifuged at $14,000 \mathrm{x}$ g for $30 \mathrm{~min}$
A


Figure 1. Effects of ethanol and Y-27632 treatment on (A) transepithelial resistance (TEER) and (B) mucosal-to-serosal flux of the intestinal epithelial barrier (IEB), assessed by the Lucifer yellow (LY) flux rate. All values represent means \pm standard deviation $\left(\mathrm{SD}(\mathrm{n}=4) .{ }^{*} \mathrm{P}<0.01\right.$ vs. the 0 -min group; ${ }^{*} \mathrm{P}<0.01$ vs. the 60 -min group.

at $4^{\circ} \mathrm{C}$ as described above. The supernatant was collected as the insoluble fraction (IS). Equal amounts of proteins (40-50 $\mu \mathrm{g}$ ) were separated by SDS-polyacrylamide gel electrophoresis (PAGE), and immunoblotting was performed by incubating with antibodies targeting ZO-1 or claudin-1 (both at 1:1,000 dilution; Santa Cruz Biotechnology. Inc., Santa Cruz, CA, USA). The protein bands were visualized and quantified using the ChemiImager 5500 version 2.03 software (AlPha InnCh, Miami, FL, USA). The integrated density values (IDV) for each protein were calculated using the computerized image analysis system Fluor Chen 2.0 (Bio-Rad, Hercules, CA, USA) and were normalized to the IDV of $\beta$-actin, used as the loading control.

Immunofluorescence. The Caco- 2 cell monolayers grown on glass coverslips were fixed in $4 \%$ paraformaldehyde and permeabilized with $0.5 \%$ Triton X-100. Following blocking with $2 \%$ BSA in PBS, the cells were incubated with rabbit anti-ZO-1 (1:50 dilution) and -claudin-1 antibodies (1:100 dilution; both from Santa Cruz Biotechnology, Inc.) in order to visualize the distribution of ZO-1 and claudin-1 proteins. The glass slides were analyzed using immunofluorescence microscopy (Olympus, Japan).

Statistical analysis. Experiments were repeated at least three times. Data are expressed as mean \pm standard deviation (SD). Differences between the groups were analyzed using one-way analysis of variance (ANOVA) followed by Dunnett's tests. 

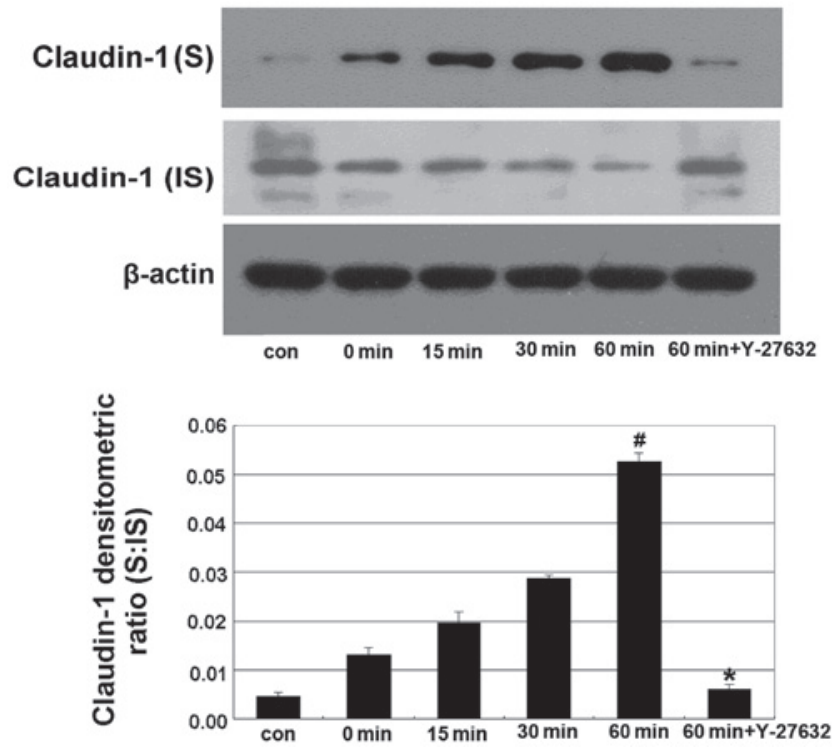

Figure 2. Effects of ethanol and Y-27632 treatment on the claudin-1 soluble (S) to insoluble (IS) ratio of integrated density values (IDV). Caco-2 cells were pretreated with Y-27632 and then treated with 5\% ethanol for $60 \mathrm{~min}$. Values represent means \pm standard deviation $(\mathrm{SD})(\mathrm{n}=4) .{ }^{*} \mathrm{P}<0.01 \mathrm{vs}$. the 0 -min group; ${ }^{*} \mathrm{P}<0.01$ vs. the 60 -min group. Con, control cells.


Figure 3. Effects of ethanol and Y-27632 treatment on zonula occludens-1 (ZO-1) protein expression, expressed as integrated density values (IDV). Caco-2 cells were pretreated with Y-27632 and then treated with $5 \%$ ethanol for $60 \mathrm{~min}$. Values represent means \pm standard deviation $(\mathrm{SD})(\mathrm{n}=4)$. ${ }^{\prime \prime} \mathrm{P}<0.01$ vs. the 0 -min group; ${ }^{*} \mathrm{P}<0.01$ vs. the 60 -min group. Con, control cells.
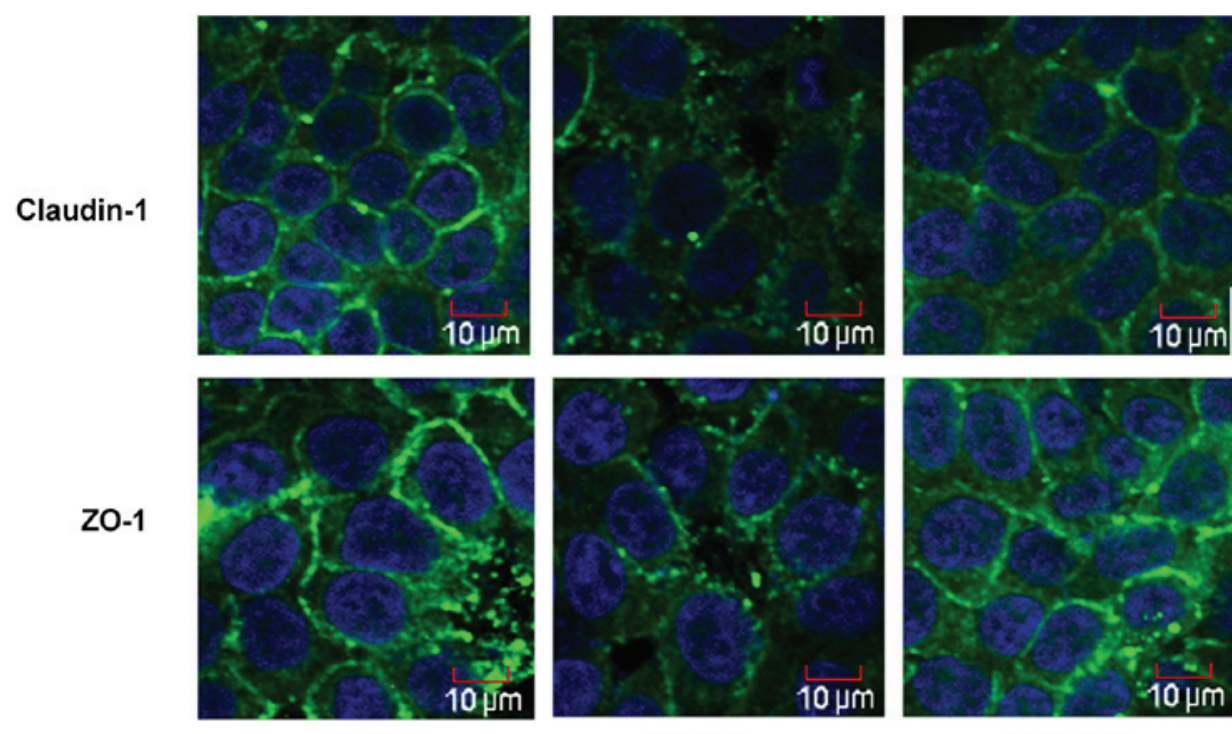

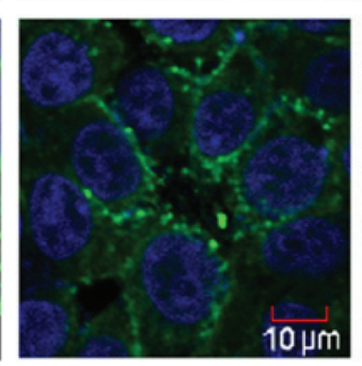

$60 \mathrm{~min}$

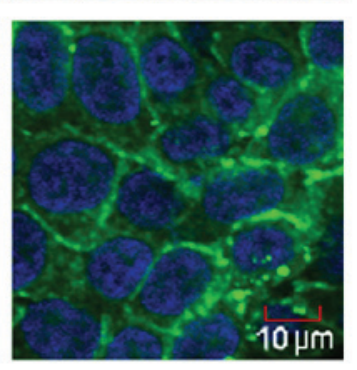

Y-27632

Figure 4. Immunofluoresence-based localization of claudin-1 and zonula occludens-1 (ZO-1) proteins in the intestinal epithelial barrier (IEB) of Caco-2 cells following ethanol treatment. Caco-2 cells of the control (con) and the 60-min groups were treated with $5 \%$ ethanol for 60 min, while the Y-27632 group was pretreated with Y-27632 and then treated with 5\% ethanol for $60 \mathrm{~min}$. Claudin-1 was relocated from the cellular membrane to the cytoplasm and the nucleus, and ZO-1 was discontinuously distributed in the boundaries of Caco-2 cells.

All statistical tests were two-sided, with $\mathrm{P}<0.05$ considered to indicate statistically significant differences.

\section{Results}

Effect of Y-27632 on ethanol-induced IEB permeability. As shown in Fig. 1, treatment of cells with 5\% ethanol induced a time-dependent decrease in TEER, with the lowest value obtained after 60 min of treatment, where this decrease was significant. A time-dependent increase in the LY flux rate as a result of ethanol treatment was also observed, with the maximum rate observed at $60 \mathrm{~min}$ of treatment, where the difference to the control cells was also significant. Y-27632 partially and significantly inhibited ethanol-induced epithelial leakage by restoring the TEER values and the LY flux rate of the IEB.

The Y-27632 inhibitor prevents the ethanol-induced shift from the insoluble to the soluble fraction of claudin-1. In this experiment, Caco-2 cells were treated with $5 \%$ ethanol for 
$0,15,30$ and $60 \mathrm{~min}$ in the alcohol-treated groups, and were pretreated with $10 \mu \mathrm{m} \mathrm{Y}-27632$ for $60 \mathrm{~min}$ prior to treatment with 5\% ethanol for $60 \mathrm{~min}$ in the Y-27632 group. We found that treatment with $5 \%$ ethanol induced a shift in the distribution of claudin-1 from the IS to the S fraction, while Y-27632 significantly reverted this shift (Fig. 2).

The Y-27632 inhibitor prevents the ethanol-induced decrease in the expression of $\mathrm{ZO}-1$. The expression of the TJ-associated protein ZO-1 displayed a progressive decline from $15 \mathrm{~min}$ of treatment with ethanol, and reached its lowest level at $60 \mathrm{~min}$. Y-27632 treatment significantly reverted this effect (Fig. 3).

The Y-27632 inhibitor affects the ethanol-induced relocalization of claudin-1 and ZO-1. The cellular distribution of ZO-1 and claudin-1 was assessed by immunofluorescence microscopy (Fig. 4). Treatment with 5\% ethanol for $60 \mathrm{~min}$ induced the relocalization of claudin-1 from the cellular membrane to the cytoplasm and the nucleus, and induced a discontinuous distribution of ZO-1 at the cellular membrane. Y-27632 strongly enhanced cortical localization of claudin-1 and ZO-1 in the IEB of Caco-2 cells (Fig. 4).

\section{Discussion}

The intestinal mucosal barrier function and integrity depend on an intact paracellular pathway, which is largely regulated by intercellular junctions, i.e. TJs, adherens junctions (AJs) and desmosomes (22). The TJs are multiprotein complexescomposed of transmembrane proteins (the claudin family, occludin, junction adhesion molecules, etc) that interact with the cytoplasmic plaque proteins (e.g., ZO-1, ZO-2 and ZO-3) (22-24). In vitro studies using the conventional two-dimensional (2D) cell culture model of intestinal cell monolayers grown on filters have shown that ethanol disrupts the epithelial TJ integrity and thereby, increases paracellular permeability $(25,26)$. Thus, disassembly of tight-junction proteins is likely a causal factor in the pathogenesis of ethanol-induced intestinal barrier dysfunctions. However, whether the ROCK inhibitor Y-27632 can inhibit the ethanol-induced increase in IEB permeability had not been investigated to date.

RhoA is a member of a subfamily of small GTPases that is involved in numerous cellular functions, including the regulation of actin filament reorganization and cell shape. The Rho-associated protein kinase (ROCK) as a downstream effector of RhoA, plays important roles in cellular processes that involve actin cytoskeletal rearrangement, including stress fiber formation, axonal growth, tumor cell invasion, and activation of platelets $(27,28)$. However, it is unknown whether this effector plays a role in Rho-dependent TJ assembly. We previously showed that ethanol induces a significant timedependent decrease in transepithelial electrical resistance (TEER) and a time-dependent increase in the LY flux rate, with the lowest and the highest values, respectively, obtained after 60 min of treatment. In this study, we found that Y-27632 can prevent the decrease in TEER and the increase in the LY flux rate (Fig. 1). LY flux and TEER assays were previously used with success to evaluate the IEB permeability (29). The ROCK inhibitor Y-27632 prevented the ethanol-induced increase in IEB permeability. We used an ethanol-treated
Caco-2 intestinal epithelial cell monolayer in vitro model along with western blot analysis to investigate whether ethanol can induce intestinal barrier dysfunction and affect the expression of the TJ-associated proteins ZO-1 and claudin-1. Our results demonstrated that ethanol induces a shift in the distribution of claudin-1, from the insoluble to the soluble fraction (Fig. 2). Furthermore, ethanol affected the expression of ZO-1 in Caco-2 cells (Fig. 3), supporting the conclusion that ethanol can affect the expression of TJ-associated proteins, disrupt the TJs and increase the permeability of the intestinal barrier. Moreover, Y-27632 treatment prevented the disruption of TJ-associated proteins ZO-1 and claudin-1 induced by ethanol treatment (Figs. 2 and 3). Immunofluorescence microscopy was further used to study the distribution of claudin-1 and ZO-1 in the cells (Fig. 4). Ethanol induced a redistribution of claudin-1 from the plasmalemma to the cytoplasm and caused a discontinuous distribution of ZO-1 at the cellular membrane. Treatment with Y-27632 partly prevented the ethanol-induced relocalization of claudin-1 and ZO-1.

In conclusion, our study showed that the ROCK inhibitor Y-27632 can prevent the ethanol-induced increase in IEB permeability and TJ assembly, suggesting that ROCK is required for the ethanol-mediated increase in IEB permeability. However, Y-27632 only partially reverted the effects of ethanol treatment in our study. We argue that this suggests the involvement of additional signaling pathways and molecules in IEB permeability.

\section{References}

1. Tsukamoto $\mathrm{H}$ : Conceptual importance of identifying alcoholic liver disease as a lifestyle disease. J Gastroenterol 42: 603-609, 2007.

2. Gramenzi A, Caputo F, Biselli M, et al: Review article: alcoholic liver disease - pathophysiological aspects and risk factors. Aliment Pharmacol Ther 24: 1151-1161, 2006.

3. Adachi M and Brenner DA: Clinical syndromes of alcoholic liver disease. Dig Dis 23: 255-263, 2005.

4. Mann RE, Smart RG and Govoni R: The epidemiology of alcoholic liver disease. Alcohol Res Health 27: 209-219, 2003.

5. Adachi Y, Moore LE, Bradford BU, Gao W and Thurman RG: Antibiotics prevent liver injury in rats following long-term exposure to ethanol. Gastroenterology 108: 218-224, 1995.

6. Bode C, Kugler V and Bode JC: Endotoxemia in patients with alcoholic and non-alcoholic cirrhosis and in subjects with no evidence of chronic liver disease following acute alcohol excess. J Hepatol 4: 8-14, 1987.

7. Forsyth CB, Voigt RM, Shaikh M, Tang Y, Cederbaum AI, Turek FW and Keshavarzian A: Role for intestinal CYP2E1 in alcohol-induced circadian gene-mediated intestinal hypermeability. Am J Physiol Gastrointest Liver Physiol 305: G185-G195, 2013.

8. Zima T and Kalousova M: Oxidative stress and signal transduction pathways in alcoholic liver disease. Alcohol Clin Exp Res 29 (Suppl 11): 110S-115S, 2005.

9. Farhadi A, Keshavarzian A, Ranjbaran Z, Fields JZ and Banan A: The role of protein kinase $C$ isoforms in modulating injury and repair of the intestinal barrier. J Pharmacol Exp Ther 316: 1-7, 2006.

10. Turner JR: Intestinal mucosal barrier function in health and disease. Nat Rev Immunol 9: 799-809, 2009.

11. Yu J, Liu F, Yin P, et al: Involvement of oxidative stress and mitogen-activated protein kinase signaling pathways in heat stressinduced injury in the rat small intestine. Stress 16: 99-113, 2013.

12. Rescigno M: The intestinal epithelial barrier in the control of homeostasis and immunity. Trends Immunol 32: 256-264, 2011.

13. Ulluwishewa D, Anderson RC, McNabb WC, Moughan PJ, Wells JM and Roy NC: Regulation of tight junction permeability by intestinal bacteria and dietary components. J Nutr 141: 769-776, 2011. 
14. Blaskewicz CD, Pudney J and Anderson DJ: Structure and function of intercellular junctions in human cervical and vaginal mucosal epithelia. Biol Reprod 85: 97-104, 2011.

15. Groschwitz KR and Hogan SP: Intestinal barrier function: molecular regulation and disease pathogenesis. J Allergy Clin Immunol 124: 3-20, 2009.

16. Atkinson KJ and Rao RK: Role of protein tyrosine phosphorylation in acetaldehyde-induced disruption of epithelial tight junctions. Am J Physiol Gastrointest Liver Physiol 280 G1280-G1288, 2001.

17. Ma TY, Nguyen D, Bui V, Nguyen H and Hoa N: Ethanol modulation of intestinal epithelial tight junction barrier. Am J Physiol 276: G965-G974, 1999.

18. Rao RK: Acetaldehyde-induced increase in paracellular permeability in Caco-2 cell monolayer. Alcohol Clin Exp Res 22 $1724-1730,1998$.

19. Wojciak-Stothard B and Ridley AJ: Rho GTPases and the regulation of endothelial permeability. Vascul Pharmacol 39: 187-199, 2002.

20. Walsh SV, Hopkins AM, Chen J, Narumiya S, Parkos CA and Nusrat A: Rho kinase regulates tight junction function and is necessary for tight junction assembly in polarized intestinal epithelia. Gastroenterology 121: 566-579, 2001.

21. Tong J, Wang Y, Chang B, Zhang D and Wang B: Evidence for the involvement of RhoA signaling in the ethanol-induced increase in intestinal epithelial barrier permeability. Int J Mol Sci 14: 3946-3960, 2013.
22. Tsukita S, Furuse M and Itoh M: Multifunctional strands in tight junctions. Nat Rev Mol Cell Biol 2: 285-293, 2001.

23. Laukoetter MG, Bruewer M and Nusrat A: Regulation of the intestinal epithelial barrier by the apical junctional complex. Curr Opin Gastroenterol 22: 85-89, 2006.

24. Schulzke JD and Fromm M: Tight junctions: molecular structure meets function. Ann NY Acad Sci 1165: 1-6, 2009.

25. Rao RK: Acetaldehyde-induced barrier disruption and paracellular permeability in Caco-2 cell monolayer. Methods Mol Biol 447: 171-183, 2008

26. Sheth $\mathrm{P}$, Seth A, Atkinson KJ, et al: Acetaldehyde dissociates the PTP1B-E-cadherin-beta-catenin complex in Caco-2 cell monolayers by a phosphorylation-dependent mechanism. Biochem J 402: 291-300, 2007.

27. Leung T, Chen XQ, Manser E and Lim L: The p160 RhoA-binding kinase ROK alpha is a member of a kinase family and is involved in the reorganization of the cytoskeleton. Mol Cell Biol 16: 5313-5327, 1996.

28. Bito $\mathrm{H}$, Furuyashiki $\mathrm{T}$, Ishihara $\mathrm{H}$, et al: A critical role for a Rho-associated kinase, p160ROCK, in determining axon outgrowth in mammalian CNS neurons. Neuron 26: 431-441, 2000.

29. Cui W, Li LX, Sun CM, et al: Tumor necrosis factor alpha increases epithelial barrier permeability by disrupting tight junctions in Caco-2 cells. Braz J Med Biol Res 43: 330-337, 2010. 